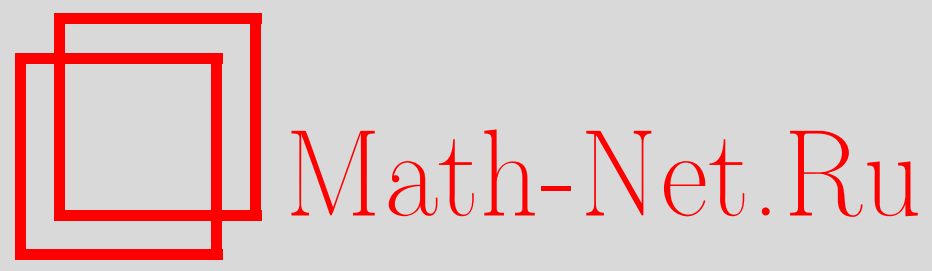

М. А. Черепнев, Схемы открытого распределения ключей на основе некоммутативной группы, Дискрет. матем., 2003, том 15, выпуск 2, 47-51

DOI: https://doi.org/10.4213/dm192

Использование Общероссийского математического портала Math-Net.Ru подразумевает, что вы прочитали и согласны с пользовательским соглашением http: //www . mathnet.ru/rus/agreement

Параметры загрузки:

IP: 18.234 .197 .8

26 апреля 2023 г., 13:22:01 


\title{
Схемы открытого распределения ключей на основе некоммутативной группы
}

\author{
() 2003 г. M. А. Черепнев
}

\begin{abstract}
В статье приведены примеры, в которых реализована высказанная В. М. Сидельниковым идея использовать некоммутативную операцию в схемах открытого распределения ключей.
\end{abstract}

Большинство известных на сегодняшний момент схем открытого распределения ключей основано на стойкости задачи дискретного логарифмирования.

В [1] В. М. Сидельниковым была высказана альтернативная идея использовать в таких схемах некоммутативную операцию. Однако конкретных примеров операций, которые могли бы обеспечить работу и стойкость системы, найдено не было. В данной статъе предложены некоторые такие примеры.

Пусть $(G, *)$ - некоммутативная полугруппа с полиномиально вычислимой ассоциативной операцией * и двумя коммутативными подполугруппами $G_{1}, G_{2}$, причем элементы из разных подполугрупп между собой не коммутируют. Предложенная в [1] схема вкратце выглядит следующим образом. Абоненты $A$ и $B$ случайным независимым друг от друга образом вырабатывают секретные элементы $a_{1} \in G_{1}, a_{2} \in G_{2}$ и $b_{1} \in G_{1}, b_{2} \in G_{2}$. Затем они передают друг другу по открытому каналу связи произведения $a_{1} * a_{2}$ и $b_{1} * b_{2}$. Общий секретный ключ абоненты $A$ и $B$ вычисляют соответственно по формулам

$$
k=a_{1} *\left(b_{1} * b_{2}\right) * a_{2}, \quad k=b_{1} *\left(a_{1} * a_{2}\right) * b_{2} .
$$

Для стойкости этой схемы необходимо, чтобы задача вычисления секретного ключа $k$ по известным произведениям $a_{1} * a_{2}$ и $b_{1} * b_{2}$ не имела для своего решения полиномиального алгоритма. В частности, трудной должна быть задача разложения на множители в полугруппе $G$.

Пример 1. Пусть

$$
a * b=a b\left(\frac{\eta(|a|)}{\mu(|b|)}\right),
$$

где $a, b$ состоят из больших простых чисел (чтобы нельзя было быстро разложить их на множители), $\eta, \mu$ - мультипликативные функции натурального аргумента, а скобки обозначают символ Якоби. Ясно, что эта операция может быть выполнена за полиномиальное время и легко проверить, что она удовлетворяет условию ассоциативности, то есть

$$
a *(b * c)=\left(\frac{\eta(|a|)}{\mu(|b c|)}\right) a\left(\frac{\eta(|b|)}{\mu(|c|)}\right) b c=\left(\frac{\eta(|a b|)}{\mu(|c|)}\right) a b c\left(\frac{\eta(|a|)}{\mu(|b|)}\right) b c=(a * b) * c
$$


Ясно, что секретная часть общего ключа $k$ - это его знак. Однако и он в данном случае может быть вычислен благодаря следующим двум свойствам рассматриваемой операции:

$$
\begin{aligned}
a * b \neq b * a & \Rightarrow a * b=-b * a, \\
a *(-b) & =-a * b .
\end{aligned}
$$

Действительно,

$$
a_{1} * a_{2} *\left(-\left(b_{1} * b_{2}\right)\right)=a_{1} * b_{1} * a_{2} * b_{2}=k .
$$

Этот недостаток можно преодолевать изменением структуры подполугрупा $G_{1}, G_{2}$ так, чтобы при $a \in G_{1}$ и $b \in G_{2}$ по $a * b$ нельзя было за полиномиальное время вычислить знак в равенстве

$$
a * b= \pm b * a
$$

Однако мы поступим иначе. Введение обобщенного символа Якоби позволяет увеличить объем секретной части.

Для некоторого простого $p$ пусть $\xi=\exp (2 \pi i / p)$. В кольце целых алгебраических чисел $K=\left\langle 1, \xi, \ldots, \xi^{p-2}\right\rangle_{\mathbf{Z}}$ кругового поля $Q(\xi)$ для простого идеала $J$ пусть $v_{J}(\cdot)-$ продолжение $J$-адического нормирования кольца $K$ на $Q(\xi), v_{p}(\cdot)-p$-адическое нормирование поля рациональных чисел $\mathbf{Q}$, а $N(J)=|(K / J)|-$ норма идеала $J$. Пусть $\alpha, \gamma \in Q(\xi), v_{\lambda}(\gamma)=0$, где $\lambda=1-\xi$. Обобщенный символ Якоби степени $p$ определяется (см. [2]) как произведение по всем простым идеалам

$$
\left(\frac{\alpha}{\gamma}\right)_{p}=\prod_{J \nmid p, \alpha}\left(\frac{\alpha}{J}\right)_{p}^{v_{J}(\gamma)},
$$

где

$$
\left(\frac{\alpha}{J}\right)_{p}=\xi^{j} \equiv \alpha^{(N(J)-1) / p} \bmod J
$$

В частности, при $p=2$ - это обычный символ Якоби и Лежандра.

Пример 2. Пусть

$$
a * b=a b\left(\frac{\eta(|a|)}{\mu(|b|)_{p}}\right)_{p}
$$

где $a, b \in Q(\xi)$, a $\eta, \mu$-мультипликативные функции теперь уже рационального аргумента такие, что $\eta(\xi)=\mu(\xi)=1$. Ассоциативность этой операции доказывается аналогично тому, как это было сделано в примере 1. Свойство (1) для этой операции не выполіняется, а свойство (2) приобретает вид

$$
a *\left(\xi^{t} b\right)=\xi^{t} a * b
$$

для любого $t \in \mathbf{N}$.

Свойства обобщенного символа Якоби хорошо известны (см. [2]). В частности, как видно из определения, выполнено свойство мультипликативности по верхнему и нижнему индексам, кроме того, верхний индекс можно брать по модулю нижнего. Выполнен также и закон взаимности, установленный Куммером. В [2] доказана следующая теорема. 
Теорема 1. Пусть

$$
\begin{array}{lll}
\alpha=1+\lambda^{2} u, & u \in Q(\xi), & v_{\lambda}(u) \geqslant 0 \\
\beta=1=\lambda v, & v \in Q(\xi), & v_{\lambda}(v) \geqslant 0 .
\end{array}
$$

Тогда

$$
\begin{aligned}
\left(\frac{\alpha}{\beta}\right)_{p}\left(\frac{\beta}{\alpha}\right)_{p}^{-1} & =\xi^{T(\xi \log \alpha \operatorname{Dlog} \beta) / p} \\
\left(\frac{\xi}{\alpha}\right)_{p} & =\xi^{T(\log \alpha) / p}
\end{aligned}
$$

где $T$-след $Q_{p}(\xi)$ над $Q_{p}, Q_{p}-$-адическое замыкание поля рачиональных чисел,

$$
\begin{aligned}
\log \alpha & =\log \left(1+\lambda^{2} u\right)=\lambda^{2} u-\frac{\left(\lambda^{2} u\right)^{2}}{2}+\frac{\left(\lambda^{2} u\right)^{3}}{3}-\ldots \in Q_{p}(\xi), \\
\operatorname{Dlog} \beta & =\frac{\beta^{\prime}}{\beta} \in Q_{p}(\xi),
\end{aligned}
$$

$\beta^{\prime}-$ формальная прочзводная по $\lambda$.

В [3] доказано следующее утверждение, являющееся следствием теоремы 1.

Следствие 1. Если $p \geqslant 3$, то

$$
\alpha, \beta \in\left\{1+x \lambda^{(p+1) / 2} \mid x \in Q(\xi), v_{\lambda}(x) \geqslant 0\right\} \Rightarrow\left(\frac{\alpha}{\beta}\right)_{p}=\left(\frac{\beta}{\alpha}\right)_{p} .
$$

Теперь ясно, что если $\eta, \mu$ принимают значения из $Q(\xi)$, то относительно операции (3) $K \backslash\{0\}$ является, вообще говоря, некоммутативной полугруппой, где 1 является двусторонней единищей.

Функции $\eta, \mu$ могут быть устроены, например, следующим образом: $f(N(a))$, где $N(a) \in \mathbf{Z}$, а $f$ - мультипликативная функция целого аргумента, заданная на простых рациональных числах равенством

$$
f(q)=\prod \alpha_{q}
$$

где справа стоит единица или произведение по некоторому фиксированному набору элементов из $Q(\xi)$, имеющих вид

$$
1 \pm \lambda^{\alpha}, \quad \alpha \in \mathbf{N}
$$

Рассмотрим символ Якоби с взаимно простыми верхним и нижним индексами такого вида. Допустим также, что показатели степени в выражении (5) для верхнего и нижнего индексов взаимно просты. Такой символ может быть вычислен не более чем за $O(\log (\max \{\alpha, \beta\})$ переворотов и взятий верхнего индекса по модулю нижнего. Действительно, эти преобразования не меняют структуру верхнего и нижнего индексов и они остаются взаимно простыми, а взятие по модулю сводится к делению с остатком одного показателя на другой. Поэтому, ввиду взаимной простоты показателей, все сведется к вычислению символа вида

$$
\left(\frac{2}{1+\lambda}\right)_{p}=\left(\frac{2}{\xi}\right)_{p}=1
$$


или

$$
\left(\frac{2}{1+\lambda}\right)_{p}=\left(\frac{2}{2+\xi}\right)_{p},
$$

который после одного переворота можно посчитать по определению, используя известные выражения для нормы и количества идеалов - делителей числа 2 (см., например, [3]).

Один переворот осуществляется за полиномиальное по $p$ время. Действительно, $T: K \rightarrow \mathbf{Z}$, при $0<i<p$

$$
\begin{aligned}
T(1) & =\sum_{j=1}^{p-1} 1=p-1 \\
T\left(\xi^{i}\right) & =\sum_{j=1}^{p-1}\left(\xi^{j}\right)^{i}=\sum_{t=1}^{p-1} \xi^{t}=-1 \\
T\left(\lambda^{i}\right) & =T\left((1-\xi)^{i}\right)=T\left(\sum_{k=0}^{i}\left(\begin{array}{l}
i \\
k
\end{array}\right) \xi^{k}(-1)^{k}\right) \\
& =\sum_{k=0}^{i}\left(\begin{array}{l}
i \\
k
\end{array}\right) T\left(\xi^{k}\right)(-1)^{k}=p-\sum_{k=0}^{i}\left(\begin{array}{l}
i \\
k
\end{array}\right)(-1)^{k}=p .
\end{aligned}
$$

Поскольку $p=\lambda^{p-1} u$, где $u \in K$ - обратимый элемент, при $i \geqslant p$ для

$$
\lambda^{i-p} u=a_{0}+a_{1} \lambda+\ldots+a_{p-2} \lambda^{p-2} \in K
$$

при $a_{k} \in \mathbf{Z}, k=0,1, \ldots, p-2$, получим, что

$$
\begin{aligned}
T\left(\lambda^{i}\right) & =p T\left(a_{0} \lambda+a_{1} \lambda^{2}+\ldots+a_{p-1} \lambda^{p-1}\right) \\
& =p\left(a_{0} T(\lambda)+a_{1} T\left(\lambda^{2}\right)+\ldots+a_{p-1} T\left(\lambda^{p-1}\right)\right)=p^{2} q_{i}, \quad q_{i} \in \mathbf{Z} .
\end{aligned}
$$

Поэтому $p^{2}$ делит $T\left(\lambda^{i}\right)$ при $i \geqslant p$, то есть формула для вычисления закона взаимности не зависит от степеней, больших $p-1$.

Таким образом, при небольших значениях $p$ операция (3) эффективно вычисляется, в то время как соответствующее разложение методом полного перебора возможных сомножителей требует экспоненциального по $p$ времени (каждый сомножитель может, вообще говоря, иметь $p$ коэффициентов).

Коммутирующие множества могут быть построены при помощи следствия 1 или с использованием свойства (4). Пусть

$$
a * b=\xi^{t} b * a, \quad a * c=\xi^{s} c * a .
$$

Тогда для $d=b * \ldots * b * c * \ldots * c$, где число элементов $b$ равно $x$, а число элементов $c$ равно $y$, справедливо соотношение

$$
a * d=d * a \Longleftrightarrow x t+y s \equiv 0 \quad(\bmod p) .
$$

Последнее сравнение при любых фиксированных $s, t$ дает как минимум $p$ различных по модулю $p$ пар $(x, y)$.

При использовании больших $p$ подсчет обобщенного символа Якоби в общем случае очень трудная задача. В некоторых случаях ее решение дает решение задачи дискретного логарифмирования (см. [3]). Однако для верхних и нижних индексов специального вида она может оказаться простой. 


\section{Список литературы}

1. Сидельников В. М., Черепнев М. А., Ященко В. В., Системы открытого распределения ключей на основе некоммутативных полугрупп. Докл. АН СССР (1993) 332, №5, 566-567.

2. Artin E., Tate J., Class field theory. Harvard Univ. Press, Harvard, 1961.

3. Adleman L. M., Pomerance C., Rumely R. S., On distinguishing prime numbers from composite numbers. Ann. Math. (1983) 117, 173-206.

Статья поступила 10.06.2002. 